\title{
Toma de decisiones en estudiantes de Grado de Ciencia Política y Administración Decision-making in Political Science and Administration degree students
}

\author{
Nuria Rodríguez Ávila ${ }^{1}$, Maria Carme Riera i Prunera ${ }^{1}$ \\ nrodriguez@ub.edu, mcriera-prunera@ub.edu \\ ${ }^{1}$ Departamento de Sociología y Análisis de las \\ Organizaciones \\ Universitat de Barcelona \\ Barcelona, Spain \\ ${ }^{2}$ Departamento de Econometría, Estadística y \\ Economía Aplicada \\ Universitat de Barcelona \\ Barcelona, Spain
}

\begin{abstract}
Resumen- La toma de decisiones es una de las competencias principales de los graduados en Ciencia Política y de la Administración. Analizamos una encuesta realizada a estudiantes durante los cursos 2006-2007 al 2012-2013, para la asignatura de Estructura Social de carácter obligatorio y equivalente a 6 créditos ECTS. Aplicamos el análisis multivariante para reducir la dimensionalidad de las variables estudiadas. Se construyen cuatro factores y un modelo de regresión logístico multinomial. Los resultados obtenidos indican que tres de los cuatro factores influyen positivamente a la hora de incrementar la probabilidad de pasar de una capacidad de Toma de decisiones de nivel nulo a un nivel elevado, sólo dos si se pasa a un nivel intermedio, y únicamente uno cuando se pasa a un nivel bajo.
\end{abstract}

Palabras clave: Competencias, Tomas de decisiones, Análisis factorial, Grado de Ciencia Política y Administración.

Abstract- Decision making is one of the main competencies graduates in Political Science and Administration should achieve. We analyzed a survey conducted among students from the 2006-2007 to the 2012-2013 academic courses, for the subject Social Structure, a compulsory subject with 6 ECTS credits. We used a multivariate analysis to reduce the dimensionality of the variables. Four factors were retained and then we applied a multinomial logistic regression model. The results indicate that (1) three of the factors have a positive influence on the probability to move from a null to a large decision making ability, and (2) the higher the decision making capacity, the more factors end up exerting a positive influence.

Keywords: Competences, Decision Making, Factor Analysis, Degree of Political Science and Administration.

\section{DESCRIPCIÓN}

La toma de decisiones es una de las competencias centrales en el grado de Ciencias Políticas y de la Administración de la Universidad de Barcelona. Esta competencia es analizada y estudiada por la sociología desde áreas como las organizaciones y las profesiones dada su influencia en la vida profesional, social y privada, así como el hecho que cada vez es más necesario ampliar conocimientos y adaptarse a las nuevas situaciones complejas. Esto ha supuesto que se incremente la necesidad de expertos con capacidad de tomar decisiones, y en el caso que nos ocupa, los graduados en Ciencias Políticas y Administración, deben tomar decisiones en el ejercicio de su profesión, así como dar o proponer soluciones a los problemas y conflictos que se presentan y generan en su trabajo habitual. En este sentido desde hace tiempo se observa cómo se produce un incremento de las áreas sociales en las que aparecen problemas que requieren de la acción de los expertos (Rodríguez, 2008).

La toma de decisiones consiste en la capacidad de decidir ante una situación donde se presentan diferentes alternativas posibles en la que intervienen la capacidad de análisis, de evaluación y comparativa. Un aspecto relevante de la toma de decisiones es la calidad de las mismas, puesto que determinará que estas sean óptimas. A su vez, esta competencia está altamente relacionada con otras, como son la capacidad de analizar la información, y comunicación.

Las teorías sobre organizaciones se preocupan ampliamente sobre la toma de decisiones. Entre ellas destaca la Teoría de la racionalidad limitada de March y Simon, que enfatiza la construcción del "El mito del buen gestor" y donde los autores analizan cómo los gestores o tomadores de decisiones en condiciones ideales se ajustarían a la toma de decisiones más adecuada en comparación a cómo realmente se toman las decisiones, así como las limitaciones que se presentan en la gestión real (ver tabla 1). Esta teoría trabaja el concepto de Certeza en la que se toman las decisiones, entendiendo como certeza aquello que se sabe que sucederá seguramente. El segundo concepto que trata son las situaciones de riesgo donde el tomador de decisiones es sabedor de los riesgos porque conoce las posibles situaciones y sabe cuál es la más acertada. Por último están las situaciones de incerteza, donde el tomador desconoce determinados aspectos de la toma de decisiones. Es por todo ello que March y Simon parten de la idea de que las decisiones (1) se toman por una serie de alternativas y análisis de la realidad a través del método de ensayo y error, (2) se opta por la más aceptable en términos políticos, y, por último (3) por inercia: siguiendo rutinas, tradición (March y Simon; 2005) 
Tabla 1. Toma de decisiones

\begin{tabular}{|c|c|}
\hline IDEAL & REAL \\
\hline $\begin{array}{l}\text { 1. Planificar estrategias a largo } \\
\text { plazo }\end{array}$ & $\begin{array}{l}\text { 1. Planificar a largo plazo está lleno de } \\
\text { incertidumbres }\end{array}$ \\
\hline $\begin{array}{l}\text { 2. Fijar objetivos y prioridades a } \\
\text { corto plazo }\end{array}$ & $\begin{array}{l}\text { 2. El objetivo depende de metas } \\
\text { grupales y particulares }\end{array}$ \\
\hline $\begin{array}{l}\text { 3. Conocer todas las opciones } \\
\text { posibles }\end{array}$ & $\begin{array}{l}\text { 3. No conocen todas las alternativas } \\
\text { posibles }\end{array}$ \\
\hline $\begin{array}{l}\text { 4. Analizar su viabilidad y calcular } \\
\text { sus costes }\end{array}$ & $\begin{array}{l}\text { 4. Falta de información, medios, o } \\
\text { tiempo para cálculos }\end{array}$ \\
\hline $\begin{array}{l}\text { 5. Decidir la opción mejor (decisión } \\
\text { racional) }\end{array}$ & $\begin{array}{l}\text { 5. Nunca se está seguro de cuál es la } \\
\text { opción mejor }\end{array}$ \\
\hline 6. Diseñar un plan de acción & $\begin{array}{l}\text { 6. Se desconocen las consecuencias de } \\
\text { los actos }\end{array}$ \\
\hline $\begin{array}{l}\text { 7. Implementar: control, resolver } \\
\text { problemas y conflictos }\end{array}$ & $\begin{array}{l}\text { 7. Empleados y otras agencias tienen sus } \\
\text { propias metas }\end{array}$ \\
\hline 8. Evaluar el logro de los objetivos & 8. Es difícil medir el cambio \\
\hline $\begin{array}{l}\text { 9. Aplicar lo aprendido a nuevos } \\
\text { proyectos }\end{array}$ & $\begin{array}{l}\text { 9. Las condiciones de cada experiencia } \\
\text { son irrepetibles }\end{array}$ \\
\hline
\end{tabular}

Fuente: (Perrow, C,1990)

Competencias del grado de Ciencia Política y de la Administración en la Universidad de Barcelona

El grado de Ciencia Política y de la Administración de la Universidad de Barcelona tiene como objetivo principal el análisis del poder, el sistema político y el Estado de la Administración. El grado busca formar profesionales que realicen actividades relacionadas con el mundo de la política y donde sus actividades fundamentales sean analizar y dar soporte en la toma de decisiones a partir del estudio detallado de las situaciones. El grado busca que estos profesionales se coloquen en el mercado laboral dotándolos de conocimiento empírico y práctico en los sectores en que está vinculada la política. La primera promoción de Grado de Ciencia Política de la Universidad de Barcelona se inició en el curso académico 2009-2010 (UB, Memoria Verifica). Las competencias que forman parte del plan de estudios del Título de Grado de Ciencia Política y de la Administración de la UB están regidas por las competencias profesionales definidas por la ANECA y las definidas en el proyecto Tuning (Alcañiz et al. 2013, 2014, González J, \& Wagemaar R.; 2003).

Entre las competencias que están incorporadas en el grado está por un lado el compromiso ético, donde se valora que el estudiante tenga capacidad de crítica y autocrítica de sus posicionamientos y trabajos. Para ello es necesario que desarrolle y muestre actitudes de forma coherentes en cuanto a las concepciones éticas y deontológicas del ejercicio profesional (Paadi, K,. 2014; Teijeiro. M, 2013)

Entre los ámbitos en los que estos futuros profesionales participan destacan, en principal lugar, las Instituciones Políticas y Administraciones Públicas, Organizaciones de grupos de interés y no gubernamentales, sector privado, enseñanza, investigación. Uno de los ámbitos importantes son los Organismos Internacionales y servicios exteriores del Estado (ANECA, 2008). Según la ANECA los perfiles profesionales de estos graduados son Técnicos de las Administraciones Públicas, Gestor de organizaciones, Analista de políticas públicas, Agente de desarrollo local, Analista político, Asesor y Consultor, Consultor internacional, Docente e Investigador.

\section{CONTEXTO}

\section{A. Objetivos}

- Valorar la competencia de toma de decisión de los estudiantes.

- Obtener información sobre cómo se valoran las nuevas formas de trabajo colaborativo entre estudiantes, para alcanzar las competencias definidas en los planes docentes.

- Comparar las diferentes cohortes de estudiantes en cuanto a la forma de adquirir las competencias en el proceso de formación.

\section{DESCRIPCIÓN DE LA MUESTRA Y CUESTIONARIO}

La población sujeto de estudio han sido los estudiantes del grado de Ciencia Política y Administración de la Universidad de Barcelona, durante los cursos 2006-2007 a 2012-2013, para la asignatura de Estructura Social de carácter obligatorio y equivalente a 6 créditos ECTS. Las edades van de los 17 a los 68 años, aunque el $66 \%$ de la muestra está comprendido entre los 18 y los 19 años. La distribución por género es muy igualitaria, el 50,2 son hombres y el 49,8 mujeres ( $N=442)$. En cuanto a la lengua materna, para el 55,9\% de los encuestados es el catalán, mientras que el 43,9 declara tener otra lengua como materna. Además, el 94\% de los entrevistados declara que su entrada en la universidad ha sido a través de las pruebas PAU, un 4,1\% lo hace a través de la Formación Profesional y el $1,8 \%$ restante tiene otras procedencia, básicamente mayores de 25 o 45 años.

En cuanto a los SISI, es decir, los que declaran que estudian y trabajan, representan un 35,6\% del total. De los que declaran que trabajan, lo hacen a tiempo completo un 8,3\%, a media jornada un 10,8\%, mientras que los restantes, trabajan los fines de semana o lo hacen de forma esporádica. Además, los estudiantes eligen principalmente el turno de mañana (55\%) a lo largo de todos los años académicos analizados.

La técnica de muestreo seleccionada fue la de muestreo no probabilístico por conveniencia o también conocida como muestreo incidental (Buendía, 1994). Se escoge esta técnica dada la accesibilidad y proximidad de los sujetos seleccionados por los asistentes a clase el día que se suministró el cuestionario.

El cuestionario utilizado para este estudio consta de 70 preguntas que recogen todas las competencias tanto transversales como específicas adquiridas en el proceso de aprendizaje, así como la actitud que tiene el estudiante ante dicho proceso, tal y como queda recogido en el proyecto Tuning, aunque adaptado al estudio sobre el grado de Ciencia Política (Rodriguez, 2013). Dicho instrumento consta de cuatro bloques. El primero corresponde a aspectos sociodemográficos, el segundo tiene que ver con valoración de las habilidades, el tercero está en relación con la adquisición de competencias transversales (i.e. capacidad de liderazgo, capacidad de resolución de problemas, trabajo en equipo...). Por último, el cuarto bloque es el referido a actitudes a la hora 
de estudiar. Las variables estudiadas se han medido en una escala de Likert de 1 a 4, donde 4 indica un grado elevado de adquisición (Rodríguez, 2017).

\section{Metodología y Resultados}

La metodología aplicada en este trabajo utiliza el análisis multivariante y los modelos de regresión logit multinomial. En primer lugar, a partir de los datos recogidos, se ha realizado un análisis factorial mediante componentes principales que nos permite reducir la dimensionalidad de los datos y agrupar los 15 aspectos estudiados de manera homogénea. El objetivo es encontrar el mínimo número de dimensiones que expliquen la máxima información contenida en los datos. Posteriormente, a partir de la información de los factores extraídos, se ha construido una regresión logit multinomial para analizar en profundidad la capacidad de toma de decisiones de los estudiantes del Grado Ciencia Política y de la Administración. El valor del alfa de Cronbach para la fiabilidad de cuestionario es 0'796, indicando, una elevada fiabilidad.

\section{Análisis factorial de las competencias transversales}

La prueba de medida de Kaiser-Meyer-Olkin (KMO) ofrece un valor de 0.891, por lo que rechazamos la hipótesis nula que establece que la matriz de correlaciones sea igual a la matriz identidad, y por tanto, existe correlación entre las variables; en consecuencia tiene sentido agruparlas en factores. Se incorporan un total de 15 variables que finalmente se reagrupan en los 4 factores extraídos con autovalores ${ }^{1}$ superiores a la unidad que permiten explicar el 55,99\% del total de la varianza de los datos.

Tabla 2. Análisis de componentes principales. Varianza total explicada

\begin{tabular}{|c|c|c|c|c|c|c|c|c|c|}
\hline \multicolumn{2}{|c|}{ Componente } & \multicolumn{3}{|c|}{ Autovalores iniciales } & \multicolumn{3}{|c|}{$\begin{array}{l}\text { Sumas de extracción } \\
\text { de cargas al cuadrado }\end{array}$} & \multicolumn{2}{|c|}{$\begin{array}{c}\text { Sumas de rotación } \\
\text { de cargas al } \\
\text { cuadrado }\end{array}$} \\
\hline & Total & $\begin{array}{c}\% \text { de } \\
\text { varianza }\end{array}$ & $\begin{array}{c}\% \% \\
\text { acumulado }\end{array}$ & Total & $\begin{array}{c}\text { \% de } \\
\text { varianza }\end{array}$ & $\begin{array}{c}\% \\
\text { acumulado }\end{array}$ & Total & $\begin{array}{c}\text { \% de } \\
\text { varianza }\end{array}$ & $\begin{array}{c}\% \\
\text { acumulado }\end{array}$ \\
\hline 1 & 5,086 & 33,907 & 33,907 & 5,086 & 33,907 & 33,907 & 2,656 & 17,704 & 17,704 \\
\hline 2 & 1,169 & 7,793 & 41,699 & 1,169 & 7,793 & 41,699 & 2,536 & 16,905 & 34,610 \\
\hline 3 & 1,116 & 7,441 & 49,140 & 1,116 & 7,441 & 49,140 & 1,825 & 12,163 & 46,773 \\
\hline 4 & 1,029 & 6,859 & 55,999 & 1,029 & 6,859 & 55,999 & 1,384 & 9,226 & 55,999 \\
\hline
\end{tabular}

Fuente: elaboración propia a partir del estudio VIOPES

Los factores que se identifican son los siguientes: el factor 1, que agrupa 6 competencias, y al que hemos llamado emprendedor e innovador, el factor 2, que consideramos gestión del conocimiento, dado que incorpora variables como análisis y síntesis, el factor 3 , que recogería la capacidad de trabajo en equipo, y, por último, el factor 4, al que hemos llamado políglota, dado que recoge el conocimiento de idiomas y la internacionalización.
Tabla 3. Componentes principales.

Matriz de componentes rotados.

\begin{tabular}{|c|c|c|c|c|}
\hline & \multicolumn{4}{|c|}{ Componente } \\
\hline & 1 & 2 & 3 & 4 \\
\hline \multicolumn{5}{|l|}{ Factor 1. Emprendedor e innovador } \\
\hline H13. Capacidad de generar nuevas ideas (creatividad) & ,769 & ,218 &,- 077 & ,148 \\
\hline H12. Capacidad de adaptación a nuevas situaciones & ,663 & 245 & 197 & ,072 \\
\hline H26. Iniciativa y espíritu emprendedor & ,610 & ,073 & ,209 &, 264 \\
\hline H20. Capacidad de comunicarse &, 586 & ,279 & 146 & ,317 \\
\hline H27. Compromiso ético & ,508 & 187 & ,304 &,- 056 \\
\hline $\begin{array}{l}\text { H21. Valoración de la diversidad y la } \\
\text { multiculturalidad }\end{array}$ &, 460 & ,148 &, 427 &,- 359 \\
\hline \multicolumn{5}{|l|}{ Factor 2. Gestión de conocimiento } \\
\hline H2. Capacidad de aplicar conocimientos a la práctica & ,144 & ,747 & ,068 & ,134 \\
\hline H1. Capacidad de análisis y síntesis & ,189 &, 720 & ,079 & ,168 \\
\hline H9. Capacidad de aprendizaje & ,311 & ,636 & ,041 &,- 077 \\
\hline H10. Habilidades de gestión de la información & 292 & 549 & ,329 &,- 152 \\
\hline H3. Planificación y gestión del tiempo & ,009 & ,513 & ,411 & ,232 \\
\hline \multicolumn{5}{|l|}{ Factor 3. Colaboración en equipos de trabajo } \\
\hline H16. Trabajo en equipo & ,082 & ,097 & ,802 & ,187 \\
\hline $\begin{array}{l}\text { H19. Capacidad para trabajar en equipo } \\
\text { interdisciplinario }\end{array}$ & ,320 & 147 & 672 & ,148 \\
\hline \multicolumn{5}{|l|}{ Factor 4. Poliglota y comunicador } \\
\hline H6. Conocimiento de una segunda lengua & ,206 & 042 & 193 & ,753 \\
\hline H5. Comunicación oral y escrita en la propia lengua & ,223 & ,458 & ,133 &, 550 \\
\hline
\end{tabular}

Fuente: Elaboración propia a partir del estudio VIOPES

\section{El modelo de regresión multinomial}

Tras la identificación de los factores, continuamos el análisis para ver en qué medida éstos influyen en la Toma de decisiones. El estadístico de razón de verosimilitud para el contraste de significación conjunta indica que el modelo es globalmente significativo ${ }^{2}$. Por lo que respecta a la bondad de ajuste, el pseudo- $\mathrm{R}^{2}$ de McFadden ${ }^{3}$ arroja un valor de 0,223; la prueba de Cox y Snell, 0,346; y la prueba de Nagelkerke, 0,419 , con lo que puede hablarse de un ajuste más bien bueno.

Tabla 4. Modelo logit multinomial. Estimaciones

\begin{tabular}{|c|c|c|c|c|c|c|c|c|}
\hline \multirow{2}{*}{\multicolumn{2}{|c|}{$\begin{array}{l}\text { H15. Toma de } \\
\text { decisiones }^{\mathrm{a}}\end{array}$}} & \multirow[b]{2}{*}{ B } & \multirow[b]{2}{*}{$\begin{array}{l}\text { Error } \\
\text { std. }\end{array}$} & \multirow[b]{2}{*}{ Wald } & \multirow[b]{2}{*}{ Sig. } & \multirow[b]{2}{*}{$\operatorname{Exp}(B)$} & \multicolumn{2}{|c|}{$\begin{array}{c}95 \% \text { de intervalo de } \\
\text { confianza para } \\
\operatorname{Exp(B)}\end{array}$} \\
\hline & & & & & & & $\begin{array}{l}\text { Límite } \\
\text { inferior }\end{array}$ & $\begin{array}{l}\text { Límite } \\
\text { superior }\end{array}$ \\
\hline \multirow[t]{5}{*}{ Bajo } & Intercepto & 4,692 & 1,460 & 10,327 & ,001 & & & \\
\hline & FAC1_1 & ,876 & , 540 & 2,632 & 105 & 2,402 & 833 & 6,922 \\
\hline & FAC2_1 & ,562 & ,541 & 1,079 & ,299 & 1,754 & 607 & 5,064 \\
\hline & FAC3_1 &,- 077 & ,685 &, 013 & ,910 & ,925 & ,241 & 3,547 \\
\hline & FAC4_1 & 848 & ,487 & 3,035 & ,081 & 2,335 & ,899 & 6,064 \\
\hline \multirow[t]{5}{*}{ Medio } & Intercepto & 6,489 & 1,454 & 19,930 & ,000 & & & \\
\hline & FAC1_1 & 1,445 & ,544 & 7,065 & ,008 & 4,244 & 1,462 & 12,321 \\
\hline & FAC2_1 & ,749 & ,541 & 1,917 & 166 & 2,114 & ,733 & 6,100 \\
\hline & FAC3_1 & ,532 & 682 & ,609 & ,435 & 1,703 & ,447 & 6,487 \\
\hline & FAC4_1 & 1,208 & ,491 & 6,050 & ,014 & 3,346 & 1,278 & 8,759 \\
\hline \multirow[t]{5}{*}{ Alto } & Intercepto & 6,542 & 1,454 & 20,238 & ,000 & & & \\
\hline & FAC1_1 & 2,511 & ,559 & 20,156 & ,000 & 12,321 & 4,116 & 36,880 \\
\hline & FAC2_1 & 1,512 & ,553 & 7,475 & ,006 & 4,534 & 1,534 & 13,401 \\
\hline & FAC3_1 & 948 & 690 & 1,888 & 169 & 2,581 & 668 & 9,975 \\
\hline & FAC4_1 & 1,769 & , 503 & 12,393 & ,000 & 5,868 & 2,191 & 15,715 \\
\hline
\end{tabular}

Fuente: elaboración propia a partir del estudio VIOPES

Los resultados obtenidos indican que a medida en que se incrementa la capacidad de toma de decisiones, hay más factores que influyen en la probabilidad de ese aumento. Así se observa que cuando se pasa de la categoría nula a escasa o baja el único factor relevante es el factor 4, es decir la capacidad de políglota y de comunicación. A continuación

\footnotetext{
${ }^{1}$ Los autovalores expresan la cantidad de varianza total explicada por cada factor.
}

\footnotetext{
${ }^{2} \chi^{2}=69.901$, con un p-valor asociado de 0.0000 .
${ }^{3}$ El pseudo- $\mathrm{R}^{2}$ de McFadden es una medida relativa de la bondad de ajuste.
Valores comprendidos entre 0.2 y 0.4 pueden considerarse como un buen

${ }^{2} \chi^{2}=69.901$, con un p-valor asociado de 0.0000 .
${ }^{3}$ El pseudo- $\mathrm{R}^{2}$ de McFadden es una medida relativa de la bondad de ajuste.
Valores comprendidos entre 0.2 y 0.4 pueden considerarse como un buen

$\chi^{2}=69.901$, con un p-valor asociado de 0.0000 .
${ }^{3}$ El pseudo- $\mathrm{R}^{2}$ de McFadden es una medida relativa de la bondad de ajuste.
Valores comprendidos entre 0.2 y 0.4 pueden considerarse como un buen ajuste (Hauber et al., 2016).
} aragoza, ESPAÑA

IV Congreso Internacional sobre Aprendizaje, Innovación y Competitividad (CINAIC 2017) 
cuando se comparan la capacidad de toma de decisiones nula con una capacidad media, además del factor 4 , resulta también relevante el primer factor, el relacionado con la capacidad de emprendedora y de innovación. Por último, la probabilidad de pasar de una capacidad nula a una elevada o alta, está influida por los factores 1, 4 y 2. La incorporación del segundo factor, el relacionado con la gestión del conocimiento, es importante dado que nos indica que un nivel elevado de toma de decisiones, a diferencia de los niveles bajos, parece requerir un buen nivel de conocimientos. Observamos que el factor 3, el referido a la competencia del trabajo en equipo, no aparece como relevante en ningún caso. Ello puede ser debido al hecho que en último término la toma de decisiones depende del líder, por lo que el trabajo en equipo no intervendría como factor relevante.

\section{CONCLUSIONES}

La globalización y el internet de las cosas están contribuyendo a una aceleración en la evolución de las formas de trabajo en cualquier institución, obligando a diferenciar entre ser capaz y ser competente. En este sentido, la toma de decisiones es una competencia clave en este proceso.

Así, un buen tomador de decisiones debe ser capaz de trabajar autónomamente, de innovar en los procesos e incorporar soluciones nuevas a situaciones complejas. Además el papel de los idiomas y la capacidad de emplear otras lenguas va a permitir un mayor campo de actuación.

El factor comunicativo es también importante para la toma de decisiones dado que el hecho de saber transmitir de forma clara y precisa sus conocimientos, y permitir que pueda comunicarse de manera directa y apropiada es básico para que las decisiones se tomen de la forma más eficiente.

\section{AGRADECIMIENTOS}

Los datos de este trabajo pertenecen al proyecto: " $L a$ adquisición de competencias en los grados universitarios: visión y opinión de los estudiantes, docentes y empresarios (VIOPES)", financiado por la Universidad de Barcelona en la convocatoria de proyectos de investigación en Ciencias Sociales y Humanas 2012.

Las autoras también agradecen la ayuda recibida a través del proyecto REDICE16-1562: “Necessitats i requeriments laborals i formació dels graduats. Anàlisi de les discrepàncies competencials a partir de les pràctiques empresarials", financiado por el Institut de Ciències de l'Educació (ICE), de la Universitat de Barcelona.

\section{REFERENCIAS}

Alcañiz, M., Claveria, O., \& Riera-Prunera, C. (2014). Competencias en educación superior desde tres perspectivas diferentes: estudiantes, empleadores y académicos. Revista Iberoamericana de Educación (RIE), 66(2), 1-19.

Alcañiz-Zanón, M., Riera-Prunera, C., \& Claveria-González, Ò. (2013). La formació competencial dels llicenciats en economia i empresa: una visió des del seu entorn professional. Revista d'Innovació i Recerca en Educació, 6(2), 64-85. http://doi.org/10.1344/reire2013.6.2625//

Buendía, L. (1994). Técnicas e instrumentos de recogida de datos. P. Colás y L. Buendía. Investigación educativa (pp. 201.244). Sevilla: Alfar.

Cortés González, J., Hernández Saavedra, M., Marchena Rivera, T., Marqueti Machado, M., \& Nava Galán, M. (2012). Estilos de liderazgo en jefes de Servicio de Enfermería. Enf Neurol, 12(2), 84-94.

Freire, M. J., Teijeiro ÁLvarez, M. M., \& Pais Montes, C. (2013). La adecuación entre las competencias adquiridas por los graduados y las requeridas por los empresarios. Revista de Educacion, 362, 13-41. http://doi.org/10.4438/1988-592X-RE-2011-362-151

González, J., \& Wagenaar, R. (2003). Tuning educational structures in Europe. Retrieved from http://eua.be/eua/jsp/en/upload/TUNING_Announcemen t_Closing_Conference.1084282515011.pdf, (December, $\left.1^{\text {st }}, 2016\right)$.

Hauber, A., Gonzalez, J. M., Groothuis-Oudshoorn, C., Prior, T., DA, M., Cunningham, C., Bridges, J. (2016). Statistical methods for the analysis of discrete-choice experiments: a report of the ISPOR Conjoint Analysis Good Research Practices Task Force (forthcoming). Value in Health, 19(4), 300-315. http://dx.doi.org/10.1016/j.jval.2016.04.004/nhttp://www .sciencedirect.com/science/article/pii/S10983015163029 11.

Martín Del Peso, M., Rabadán Gómez, A. B., \& Hernández March, J. (2013). Desajustes entre formación y empleo en el ámbito de las enseñanzas técnicas universitarias: La visión de los empleadores de la Comunidad de Madrid. Revista de Educacion, (360), 244-267. http://doi.org/10.4438/1988-592X-RE-2011-360-110

Mason, G., Williams, G., \& Cranmer, S. (2009). Employability skills initiatives in higher education: what effects do they have on graduate labour market outcomes? Education Economics, 17(1), 1-30.

Paadi, K. (2014). Perception on employability skills necessary to enhance human resource management. Graduates prospects of securing a relevant place in the labour market. European Scientific Journal, Special ed. (August), 129-143.

Perrow, C. N. (1991). Sociología de las organizaciones (No. 307.2 P4y 1986).

Rodríguez-Ávila, N. R. (2008). Manual de sociología de las profesiones (Vol. 343). Edicions Universitat Barcelona.

Rodríguez- Ávila, N. R., Llobet, M. P., \& Jaques, T. M. (2013) Habilidades y competencias de los graduados: Estudio del caso de estudiantes de Grado de Ciencia Política (UB) y Grado de Empresariales (UPF).

Simon, H., \& MARCH, J. (2005). Administrative behavior and organizations. Organizational Behavior 2: Essential Theories of Process and Structure, 2, 41. 
Teijeiro, M., Rungo, P., \& Freire, M. J. (2013). Graduate competencies and employability: The impact of matching firms' needs and personal attainments.
Economics of Education Review, 34, 286-295. http://doi.org/10.1016/j.econedurev.2013.01.003

UB (2014) Memoria para la verificación del Título de Grado en Enfermería por la Universidad de Barcelona.

Octubre 4-6, 2017, Zaragoza, ESPAÑA 